\title{
A Comprehensive Prosthodontic Management of Subtotal Maxillectomy
}

\author{
${ }^{1}$ Manoj Kumar Sundar, ${ }^{2} \mathrm{GP}$ Surendra Kumar, ${ }^{3}$ Shruti Lakhanpal
}

\begin{abstract}
Patient: A 21-year-old female patient was diagnosed with intraosseous mucoepidermoid carcinoma on the left side of maxilla, which was planned for subtotal maxillectomy involving the left maxillary sinus.
\end{abstract}

Discussion: Prosthodontic rehabilitation of patients with maxillectomy is a challenge as it involves restoring speech, deglutition, mastication and respiration. This clinical report describes a comprehensive prosthodontic management of a young girl treated for a subtotal maxillectomy due to intraosseous mucoepidermoid carcinoma. The prosthodontic management that was carried out right from the surgical obturator to a definitive prosthesis with a two-piece closed hollow bulb, magnet-retained obturator shows the ease and simplicity in the management of a case with subtotal maxillectomy.

Conclusion: Subtotal maxillectomy defects represent a complex challenge for the maxillofacial prosthodontist. The comprehensive technique described in this clinical report can be employed in any similar case.

Keywords: Magnet, Maxilla, Osteotomy, Palatal, Obturator, Prosthesis design, Speech, Intelligibility.

How to cite this article: Sundar MK, Kumar GPS, Lakhanpal S. A Comprehensive Prosthodontic Management of Subtotal Maxillectomy. Int J Prosthodont Restor Dent 2014;4(4):120-126.

\section{Source of support: Nil}

\section{Conflict of interest: None}

\section{INTRODUCTION}

The term maxillectomy is used by head and neck surgeons and prosthodontists to describe the partial or total removal of the maxillae in patients suffering from benign or malignant neoplasms. ${ }^{1}$ Maxillectomy defects can be categorized as limited, partial, medial, subtotal, total, radical or extended. ${ }^{1}$ The hard and soft palates are anatomical structures that have widely recognized roles

${ }^{1}$ Past Postgraduate Student, ${ }^{2}$ Professor, ${ }^{3}$ Postgraduate

${ }^{1-3}$ Department of Prosthodontics, VS Dental College and Hospital, Bengaluru, Karnataka, India

Corresponding Author: Manoj Kumar Sundar, Past Postgraduate Student, Department of Prosthodontics, VS Dental College and Hospital, Bengaluru, Karnataka, India, Phone: 04424413908 e-mail: manojs87@gmail.com in speech and deglutition. A surgical approach alone without reconstruction or obturation of the surgical defect will result in air, liquid and food escaping into the maxillary sinus and nasal cavities, causing severe speech and swallowing dysfunction with significant reduction in quality of life. ${ }^{1}$

Postoperative radiotherapy in patients undergoing maxillectomy can lead to restricted mouth opening due to the scarring and fibrosis of irradiated muscles. The condition impairs the ability to incise and masticate food, interferes with oral hygiene, and restricts access for dental procedures. It may adversely affect speech and facial appearance. It can also compromise or prevent the construction and use of removable dental prostheses or appliances. ${ }^{2}$ To improve mouth opening in such patients, various appliances are available which have been described in literature. ${ }^{2}$ This article describes the fabrication of an alternative appliance based on the principle of quadhelix. A quadhelix is an orthodontic appliance, which is used, for the expansion of maxillary arch in a horizontal plane. If this principle is used for expansion in a vertical plane, then it can be employed as a mouth-opening appliance.

Thorough pre- and postsurgical reconstructive and prosthetic treatment planning ensures best rehabilitation for a maxillectomy patient. Successful obturation depends on the volume of the defect and the positioning of remaining hard and soft tissues to be used to retain, stabilize and support the prosthesis. ${ }^{3}$ The weight of the prosthesis may hamper the retention and stability; therefore, the prosthesis must be as light as possible. Obturator designs for partial and total maxillectomy defects include open or closed hollow obturators, ${ }^{4,5}$ inflatable obturators ${ }^{6}$ and two-piece hollow obturator prostheses. Maxillofacial prosthetic reconstruction of the subtotal and total maxillectomy defects uses anatomical undercuts to augment retention.?

This clinical report describes a comprehensive prosthodontic management of a patient with subtotal maxillectomy and trismus following radiotherapy, with a two-piece hollow bulb magnet retained obturator and an alternative appliance to treat trismus based on the principle of quadhelix. 


\section{OUTLINE OF THE CASE}

\section{Diagnosis, Treatment Planning and Treatment Procedures}

A 21-year-old female patient was referred from the department of oral and maxillofacial surgery requiring a surgical stent to be fabricated for a diagnosed case of intraosseous mucoepidermoid carcinoma on the left side of maxilla, which was planned for subtotal maxillectomy involving the left maxillary sinus. The diagnostic cast was duplicated with irreversible hydrocolloid. The duplicated cast was trimmed according to preplanned surgical line of resection. Cast modification was done to fabricate a surgical obturator using autopolymerising acrylic resin (Fig. 1). Wrought wire clasps were incorporated in the surgical obturator to gain retention and stability. After the subtotal maxillectomy of the left side of maxilla was carried out, the surgical obturator helped, not only in obturating the defect but also in retaining the surgical pack, which was placed in the defect (Fig. 2). The patient was subjected to post-surgery radiation therapy. Subsequently, the patient developed trismus with a limited mouth opening of $15 \mathrm{~mm}$.

A simple and innovative appliance employing the principle of quadhelix was used to increase the mouth opening. The mouth opening appliance was fabricated with a $1 \mathrm{~mm}$ diameter wire, incorporating a quadhelix in a vertical plane. It was reinforced with acrylic plates superiorly and inferiorly. The superior and inferior acrylic plates covered the occlusal surfaces of maxillary and mandibular teeth (Fig. 3). The quadhelix was placed on the maxillary cast which is blocked out with dental plaster on the vault area. The spring was secured in position with sticky wax, and autopolymerising acrylic resin plate was fabricated by sprinkle on technique. The retentive loops of the quadhelix were incorporated into the acrylic plate. The plate covered the occlusal and the

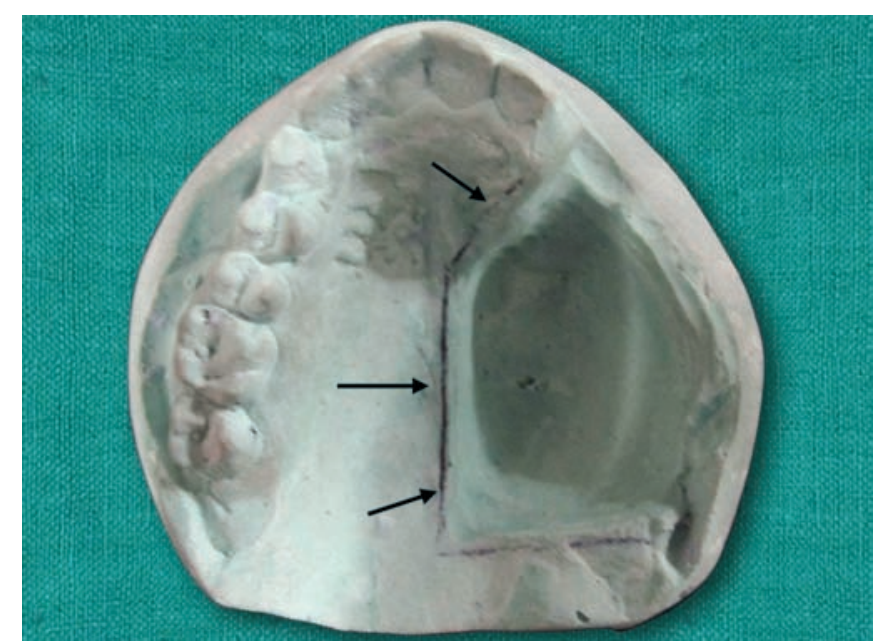

Fig. 1: Line of surgery and cast modification incisal third of the teeth. Similarly, the lower arch of the plate is fabricated. The helices were activated to gain an opening of $25 \mathrm{~mm}$ from $15 \mathrm{~mm}$. The appliance has a spring action which will provide opening forces to the jaws. The appliance is worn 5 times a day for a duration of 10 minutes for each time. Activation of the coil is done by opening the helix once a week for 4 weeks. The desired mouth opening was obtained within 2 months. And the same mouth opening was maintained during the complete course of radiation therapy. After a span of 6 months, the resected area had completely healed and the mouth opening was increased to $30 \mathrm{~mm}$.

To obturate the defect, a definitive two-piece, hollowbulb, magnet-retained obturator prosthesis was planned. Lateral scar band was noticed at the site of the defect. Following were the boundaries and communications of the defect:

- Medially, the defect wall had the nasal turbinates and was communicating to the nasal cavity.

- Posteriorly, it was communicating to the nasopharynx.

- Superiorly, the defect was bounded by the inferior wall of the orbit.

A nonfunctional impression of the defect area was carried out in two parts using addition polyvinyl siloxane putty consistency (Aquasil—Dentsply DeTrey, $\mathrm{GmbH}$ ). The first piece was indexed with three triangular notches, two toward the palatal and one toward the mesial end of the impressed putty. The second part was then adapted over the first part and the respective keys of the notches were formed, which helped in reorienting (Fig. 4). The remaining dentition and other areas were recorded with irreversible hydrocolloid impression material and the impression of the defect area was reoriented and stabilized with sticky wax (Fig. 5). Study cast was obtained, surveyed and the design of the framework was decided.

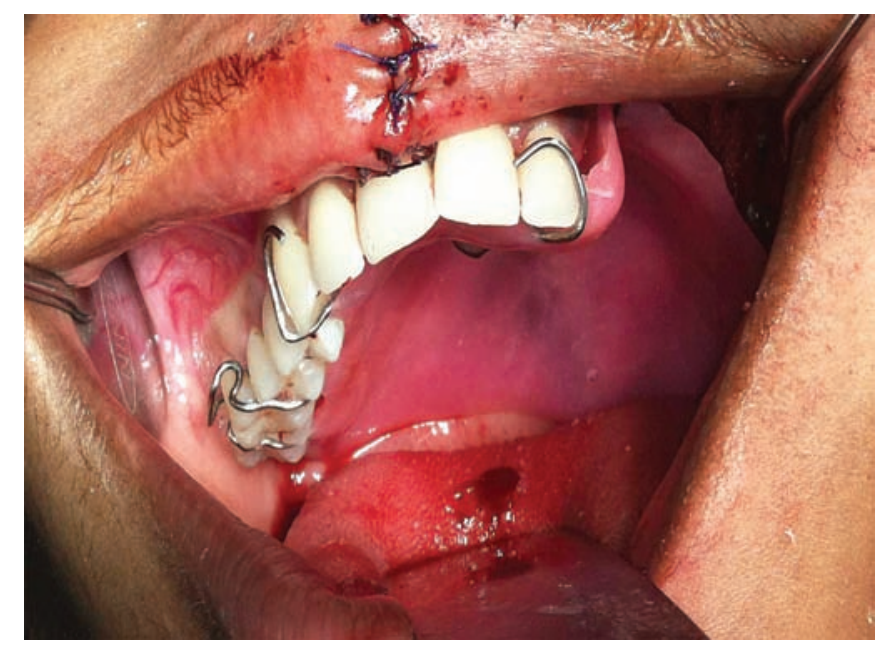

Fig. 2: Surgical obturator 


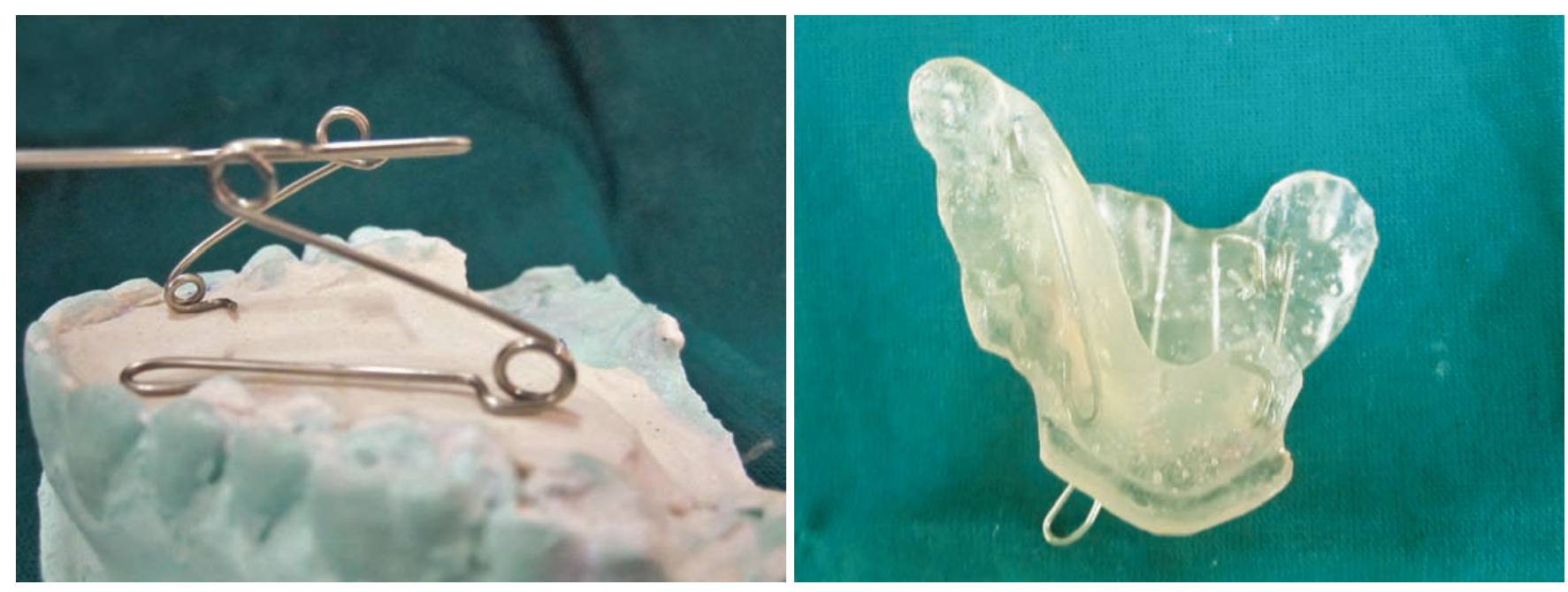

Fig. 3: Mouth opening appliance
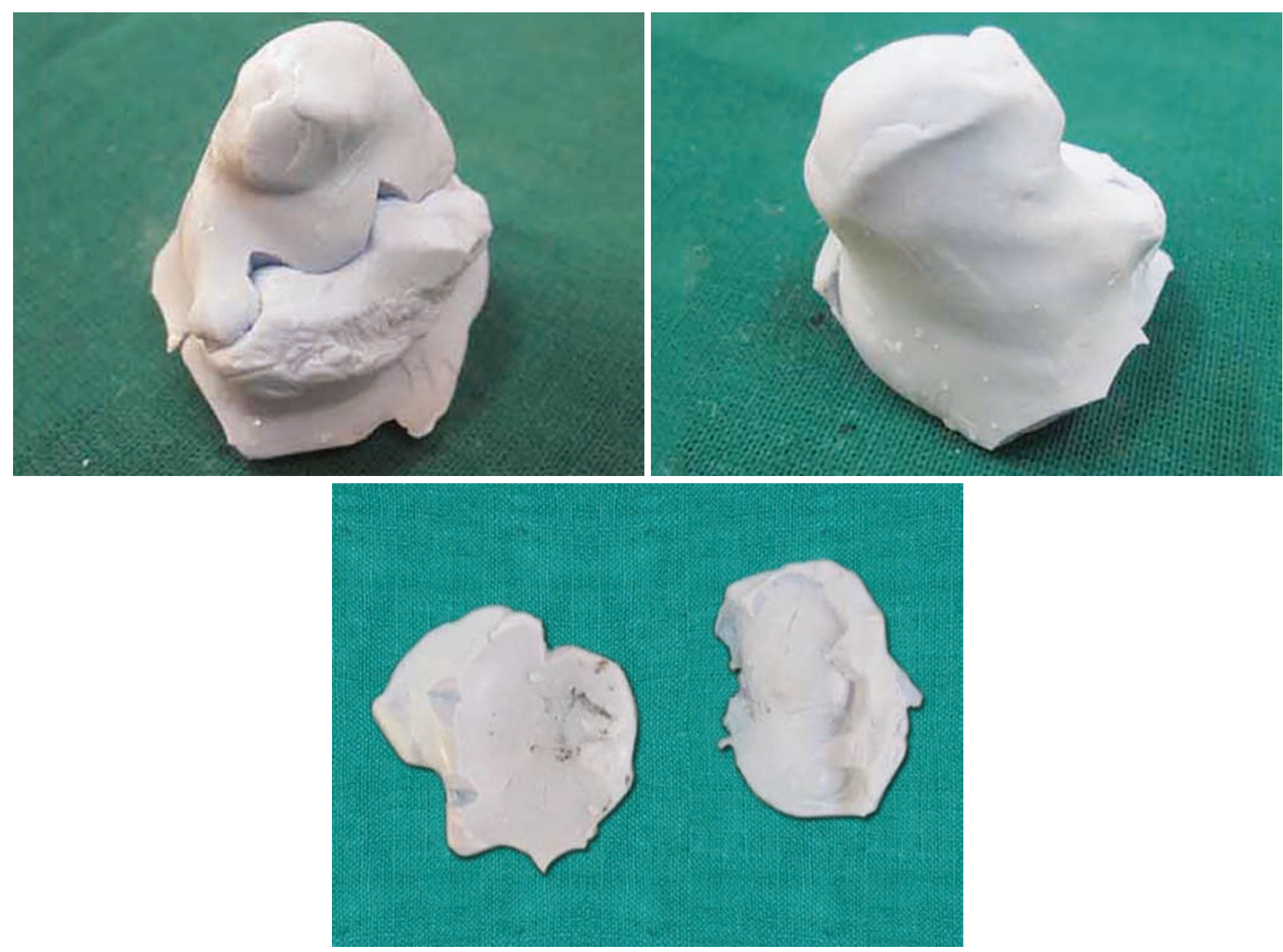

Fig. 4: Two-part putty impression of the defect

The framework design involved an embrasure clasp, a round-rest distal depression clasp and a circumferential clasp with canine extension in the form of a tripod. Lattice type of minor connector was used for the acrylic material to be held in the defect area. An anteroposterior palatal strap was used as the major connector. Preparations of the necessary rest seats were carried out. The impression of the defect was repeated in a similar manner as it was made during the preliminary impression. A definitive impression was made using addition polyvinyl siloxane medium consistency (Aquasil-Dentsply DeTrey, GmbH) in a custom acrylic tray (Fig. 6). Definitive 


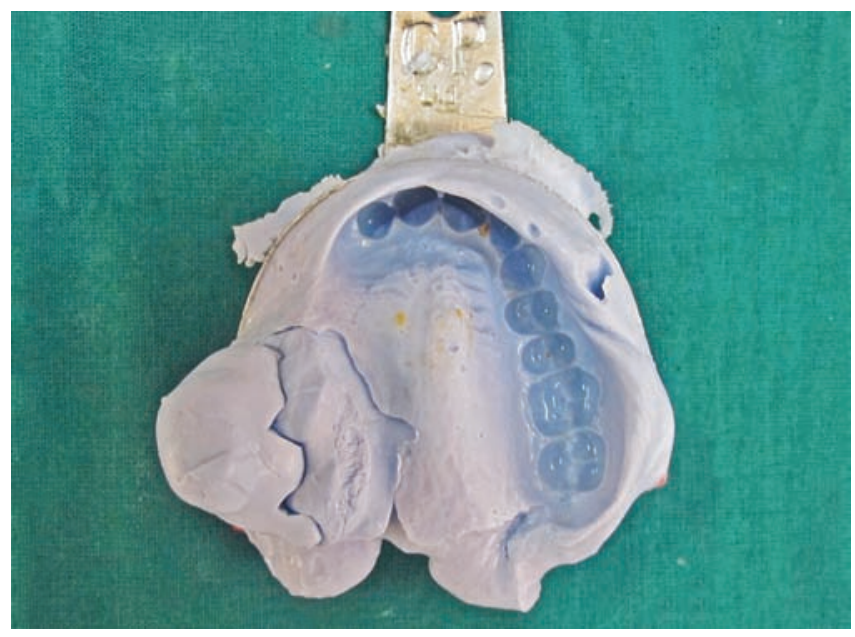

Fig. 5: Preliminary impression

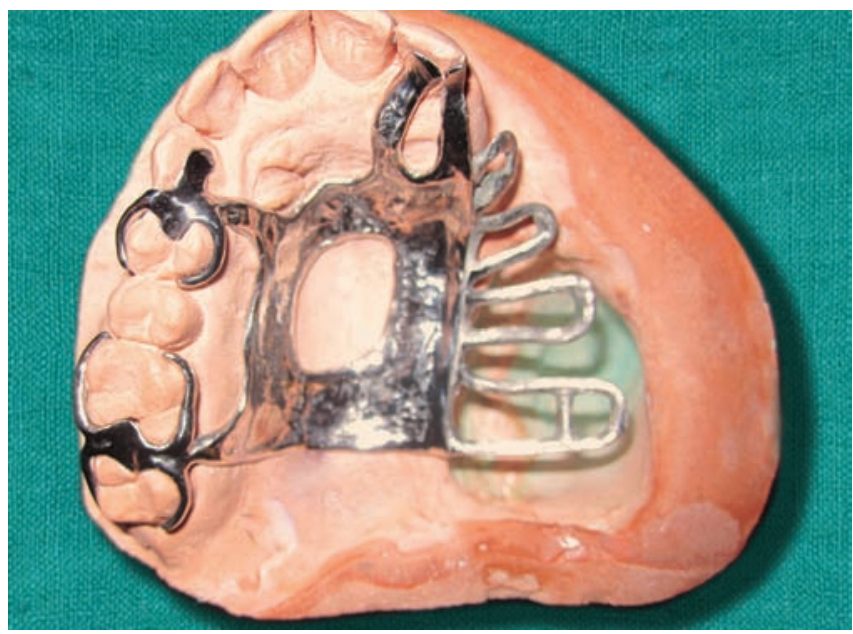

Fig. 7: Cast metal framework

cast was fabricated using type 4 die stone (Kalrock), and the framework was casted as per the design (Fig. 7). Framework trial was carried out to evaluate the fit. Wax occlusal rims were fabricated at the edentulous area on the framework and maxillomandibular relation was recorded. After teeth arrangement, the cast partial denture try-in was carried out.

To obturate the defect area, a two-piece magnetretained closed hollow bulb obturator was decided. Wax up of the defect area was done with two layers of wax sheet well adapted on the defect walls to create hollowness in the center. This was processed separately on a duplicated cast with heat-cured acrylic resin (DPI heat-cured acrylic) (Fig. 8). The open end of the bulb was closed with a thin sheet of Autopolymerising acrylic resin, into which industrial grade noncoated samarium cobalt magnet (cobalt-samarium, Ambica Corporation, New Delhi, India) was incorporated (Fig. 9). The lid was fabricated by manipulating autopolymerising acrylic resin into a sheet and by inverting the open end of the hollow bulb onto the resin sheet. The open bulb creates

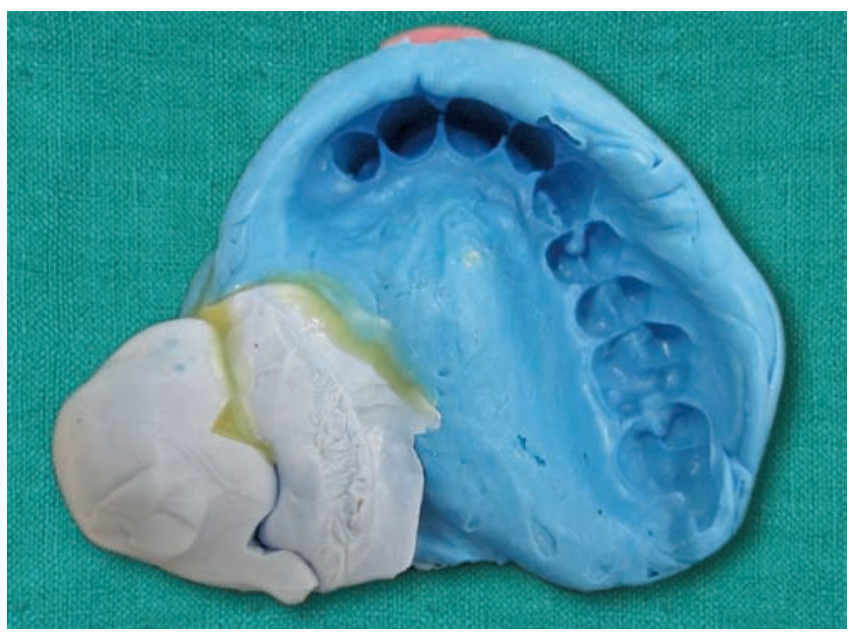

Fig. 6: Definitive impression

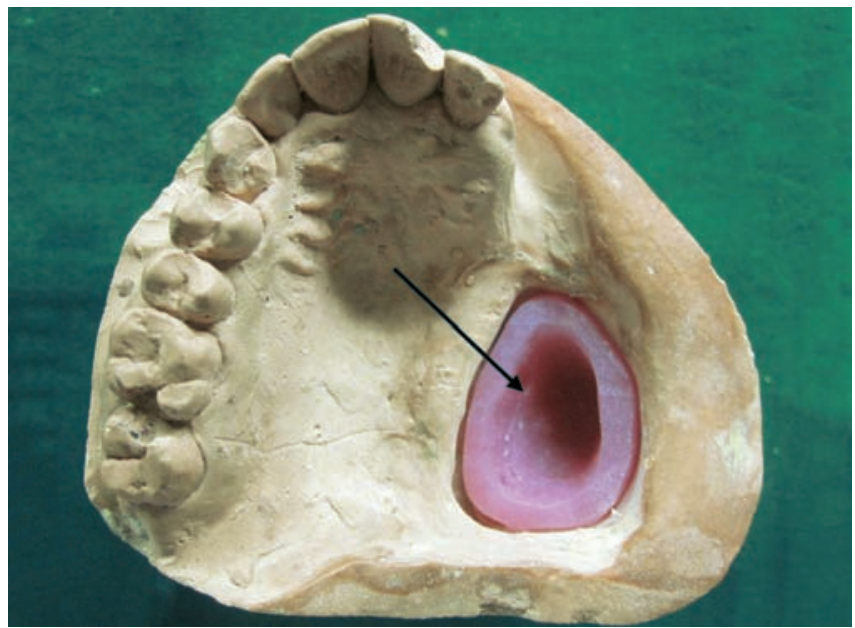

Fig. 8: Heat processed acrylic hollow bulb

a depression on the resin sheet, thereby acting as a lid. Once the resin sheet was set, it was used as a covering lid for the hollow bulb. One magnet was incorporated in the covering lid from the inner aspect by placing the magnet into the resin sheet while the material was setting. In order to have a key for the placement of the two pieces namely the closed hollow bulb and the cast partial denture, apart from the magnets, a step kind of depression was created on the flat surface of the closed hollow bulb (inferior surface). This depression created an indentation in the wax portion of the trial denture (Fig. 10). The cast partial denture was then processed with heat-cured acrylic resin separately. The denture base was made hollow by using a pear-shaped acrylic trimming bur to create a small opening distal to the most posterior tooth to remove acrylic, which was extended till the premolar region. The access hole was later covered with autopolymerizing acrylic resin (Fig. 11). The hollow bulb obturator and the processed cast partial denture were reoriented on the duplicated cast for placement and pick-up of the counter magnet in the cast partial 


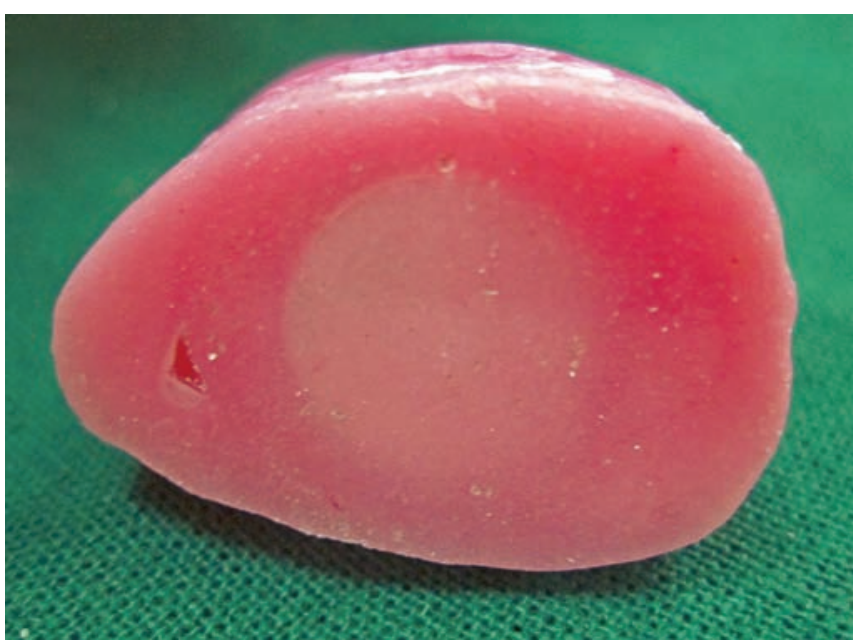

Fig. 9: Inferior flat surface incorporated with magnet covering the hollow bulb

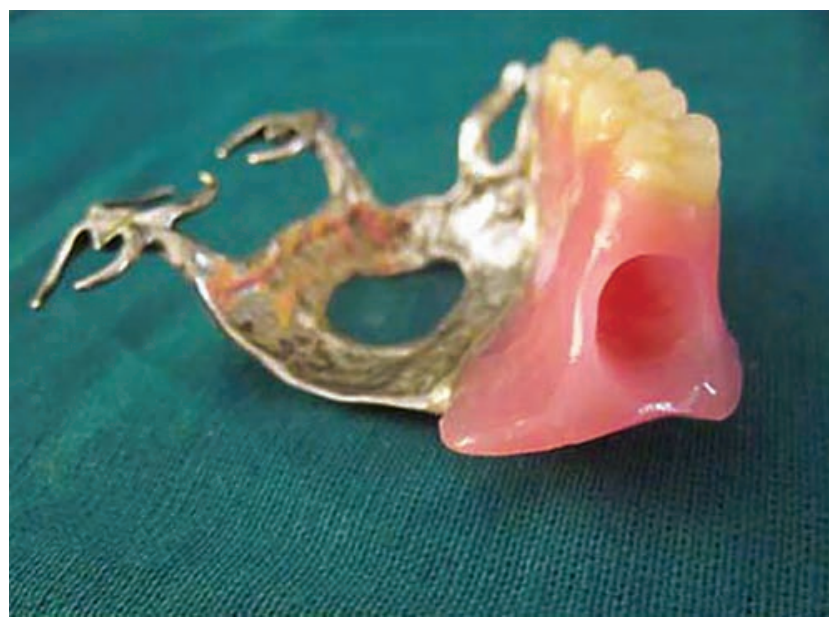

Fig. 11: Hollow denture base of cast partial denture

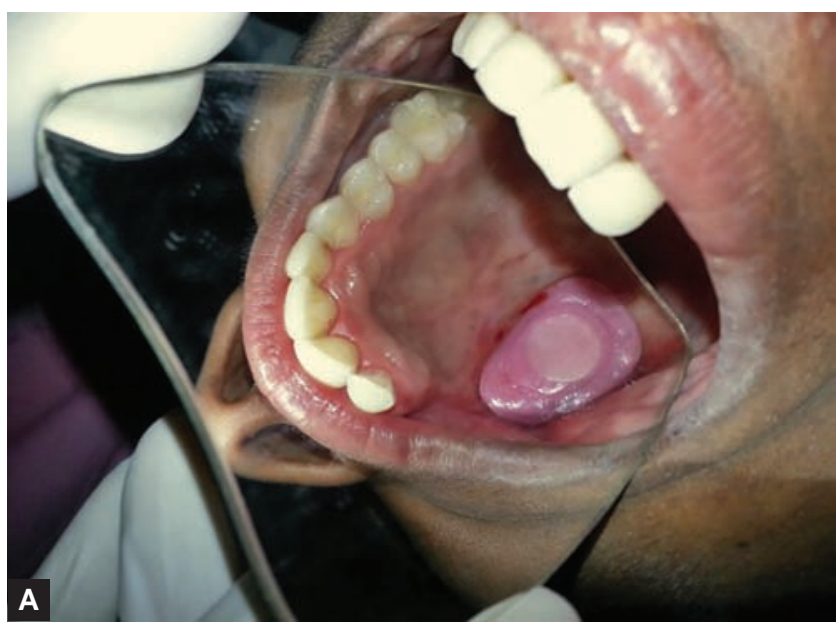

Figs 12A and B: (A) Insertion of the closed hollow bulb and (B) insertion of the cast partial denture

denture. The magnet was picked up onto the cast partial denture with the help of cyanoacrylate. First the patient was familiarized with the placement of hollow bulb obturator, by orienting the tapered basal end of the bulb anteriorly (Fig. 12A), followed by the placement of cast partial denture (Fig. 12B).
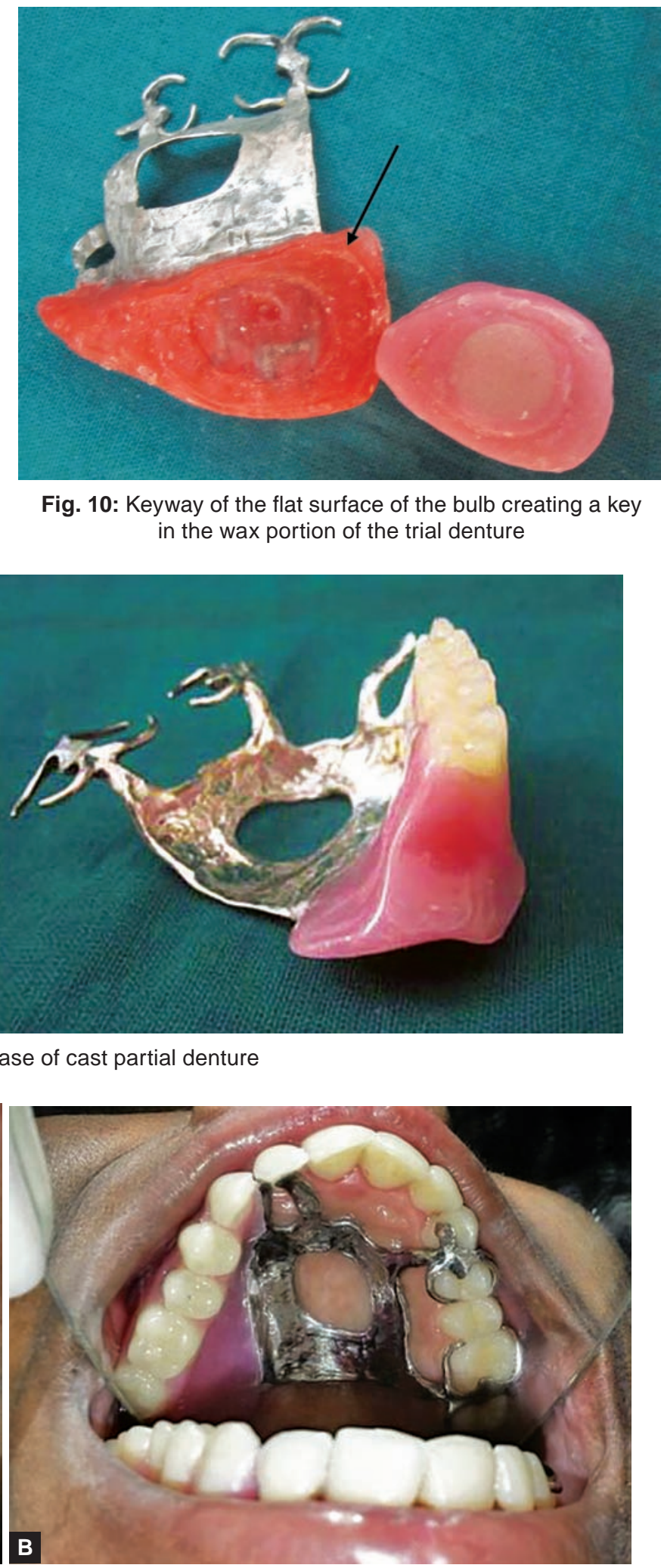

Fig. 10: Keyway of the flat surface of the bulb creating a key in the wax portion of the trial denture

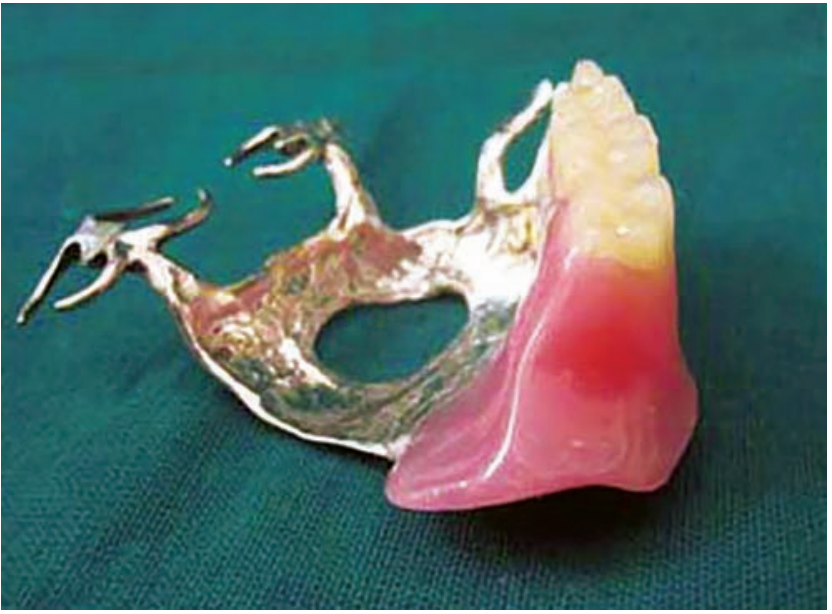

\section{Treatment Outcomes}

Postinsertion review was done after 1 week, 1 month and 8 months. Apart from slight difficulties, the patient was satisfied with the definitive prosthesis. The speech intelligibility was improved after the definitive prosthesis 


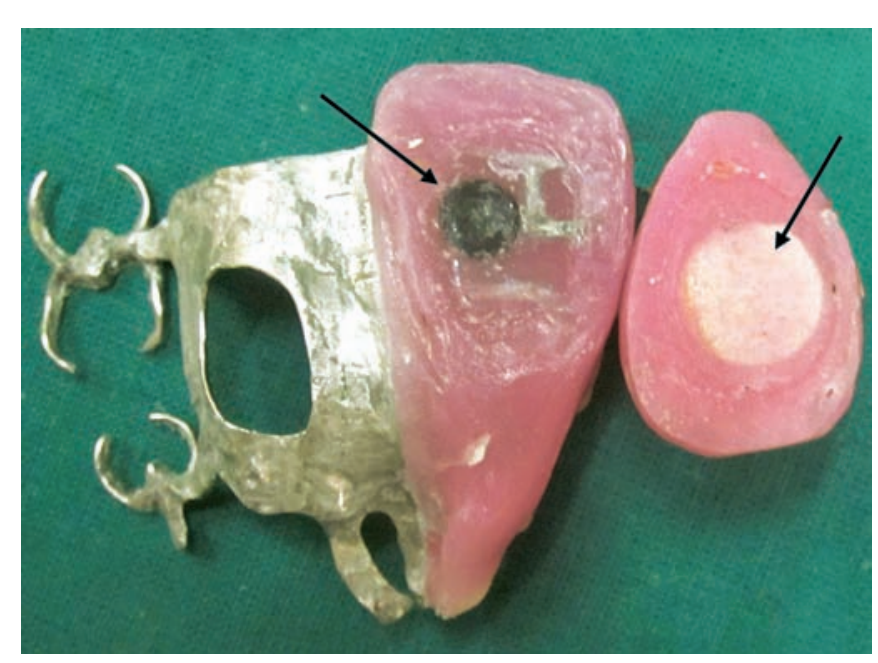

Fig. 13: Eight months post prosthetic view showing no signs of corrosion

was in place. The region of the magnets as seen after 8 months of service (Fig. 13).

\section{DISCUSSION}

Mucoepidermoid carcinoma is a tumor of the minor salivary glands. Intraosseous mucoepidermoid carcinoma is a rare entity, which involves the bone as well. 8,9

The surgical obturator, in this case, provides a scaffold for the surgical pack. The need to retain the surgical obturator for a longer period of time necessitates the requirement of additional retention. ${ }^{10}$ Hence, a tripod design with an adams clasp and two c-clasps fabricated with wrought wire were incorporated in the surgical obturator.

Postoperative radiotherapy led to fibrosis of the musculature, which resulted in a restricted mouth opening of $15 \mathrm{~mm}$. Trismus has a detrimental effect on the quality of life, because of the impairment of basic oral functions, such as chewing and swallowing. ${ }^{11}$ For a case of trismus following maxillectomy and radiation therapy, muscle exercises are the mainstay of treatment. The TheraBite Jaw Motion Rehabilitation System (TheraBite for short) is one device designed for mouth-opening exercise, and has been used for long. Some traditional simple devices including stacked tongue depressors and corkscrews have also been used in clinical practice to help mouth opening. ${ }^{12}$ However, in this case apart from prescribing home exercises to improve mouth opening, an acrylic appliance with a quadhelix was used. Activation of the appliance was carried out by opening the helix. A quadhelix is an orthodontic appliance, which is mainly used for maxillary arch expansion in a horizontal plane. However, this innovative, simple mouth-opening appliance incorporates this design in a vertical plane, in order to improve mouth opening. The mouth opening was improved to approximately to $25 \mathrm{~mm}$ and was gradually increased to $30 \mathrm{~mm}$. This appliance can be customized according to patients as the appliance fits to the incisal and occlusal third of the dental arch which improves the fitting of the appliance. Also, it is easier to construct and cost-effective.

As the resection bed was treated postoperatively with radiation therapy, it resulted in limited oral opening, and insertion of one-pieced obturator prosthesis was not possible. Thus, the treatment plan included the use of a magnet-retained two-piece hollow bulb obturator. The bulb was fabricated separately which could be fitted accurately in the patient's mouth. Providing improvement in speech was one of the priorities of the patient. Putty consistency addition silicone was used in two parts to record the impression of the defect, with a lateral wall undercut. The advantages of using it are as follows:

- It is easy to adapt and manipulate the material intraorally, and

- It is dimensionally accurate than conventional irreversible hydrocolloid.

The framework was designed to have a round-rest distal depression clasp on the lateral incisor as esthetics had to be managed without any exposure of the clasp. ${ }^{13}$ Also, the clasp design has a split minor connector, which reduces the forces on a weak abutment, like the lateral incisor.

Considering the size of the defect to be obturated, a hollow bulb obturator in this case would reduce the weight of the prosthesis. A closed hollow bulb was designed for the ease of maintenance and cleansability. However, the hollowness created in the denture base using an acrylic trimmer needs to be carried out carefully as there are chances for perforation of the denture base. Literature states various techniques of fabrication of hollow maxillary complete dentures, like using a solid three-dimensional spacer, including dental stone, cellophane wrapped asbestos, silicone putty, or modeling clay which may be effectively used in creating hollowness in the denture base. ${ }^{14}$ Also, the defect had a lateral undercut, which necessitated two different paths of insertion, one for the obturator and the other for the cast partial denture. Thus, a two-piece prosthesis was decided because the path of insertion for the obturator and the cast partial denture were different. ${ }^{15}$

The opposite polarity of the commercially available industrial grade magnets was sufficient to be incorporated in the obturator prosthesis as an additional mode of retention. A noncoated magnet exposed to an oral environment has a greater risk of corrosion. ${ }^{16,17}$ However, at 8 months follow-up, there were no clinical signs of corrosion at the site of noncoated magnets. 
The advantages of this prosthesis are that the technique is noninvasive, cost-effective, tissue tolerant, esthetic, reduced weight of the prosthesis, comfortable to use and easy to clean. Such two-piece prosthesis retained by magnets overcome the concern related to two different path of insertion as seen in this case. However, durability of surface coatings of the long-term magnets is a major concern. The disadvantage of this approach is that a few additional laboratory steps were necessary for the fabrication of the prosthesis and corrosion of magnet in oral environment, which has to be maintained well.

\section{CONCLUSION}

Subtotal maxillectomy defects represent a complex challenge for the maxillofacial prosthodontist. The comprehensive technique described in this clinical report can be employed in any similar case. In an attempt to improve the retentive and stabilizing characteristics of an obturative prosthesis, an outline of the technical construction is proposed which reduces the total weight of the appliance by processing two separate sections and creating a hollow inner chamber. The potential benefits of the technique are availability and ease of fabrication of the prosthesis with additional retention using commercially available industrial grade magnets.

\section{REFERENCES}

1. Ortegon SM, Martin JW, Lewin JS. A hollow delayed surgical obturator for a bilateral subtotal maxillectomy patient: a clinical report. J Prosthet Dent 2008;99(1):14-18.

2. Lund TW, Cohen JI. Trismus appliances and indications for their use. Quintessence Int 1993;24:275-227.

3. Watson RM, Gray BJ. Assessing effective obturation. J Prosthet Dent 1985;54(1):88-93.

4. Brown KE. Fabrication of a hollow-bulb obturator. J Prosthet Dent 1969 Jan;21(1):97-103.
5. Palmer B, Coffey KW. Fabrication of the hollow bulb obturator. J Prosthet Dent 1985;53(4):595-596.

6. Payne AG, Welton WG. An inflatable obturator for use following maxillectomy. J Prosthet Dent 1965 Jul-Aug;15: 759-763.

7. Cheng AC, Somerville DA, Wee AG. Altered prosthodontic treatment approach for bilateral complete maxillectomy: a clinical report. J Prosthet Dent 2004;92(2):120-124.

8. Browand BC, Waldron CA. Central mucoepidermoid tumors of the jaw: report of nine cases and review of the literature. Oral Surg Oral Med Oral Pathol 1975;40(5):631-643.

9. Namin AK, Moshref M, Shahoon H, Mashhadi A, Khojasteh A. Intraosseous mucoepidermoid carcinoma of the maxilla in a teenager: a case report and review of literature. Oral Surg Oral Med Oral Pathol Oral Radiol Endod 2005 Dec;100(6):E93-96.

10. Jacob FJ. Clinical management of the edentulous maxillectomy patient. In: Taylor TD, editor. Clinical maxillofacial prosthetics. Chicago: Quintessence; 2000. p. 85-87.

11. Deogade SC, Mantri SS. Rehabilitation of a partial maxillary defect with magnet retained two-piece hollow bulb obturator. Eur J Prosthodont 2014;2:62-66.

12. Ren WH, Ao HW, Lin Q, Xu ZG, Zhang B. Efficacy of mouth opening exercises in treating trismus after maxillectomy. Chin Med J (Engl) 2013 Jul;126(14):2666-2669.

13. Tran C, LaBarre E, Landesman HM. A removable partial denture using an esthetically designed round-rest distal clasp on maxillary anterior abutment teeth: a clinical report. J Prosthet Dent 2009 Nov;102(5):286-289.

14. Radke U, Mundhe D. Hollow maxillary complete denture. J Ind Prosthodont Soc 2011 Dec;11(4):246-249.

15. Oh WS, Roumanas E. Dental implant-assisted prosthetic rehabilitation of a patient with a bilateral maxillectomy defect secondary to mucormycosis. J Prosthet Dent 2006;96(2): 88-95.

16. Hatami M, Badrian H, Samanipoor S, Goiato MC. Magnetretained facial prosthesis combined with maxillary obturator. Case Reports in Dentistry 2013;Article ID 406410:5. DOI:10.1155/2013/406410.

17. Goyal S, Goyal MK, Kallingan P. Magnetically retained extraoral prosthesis and maxillary interim obturator. Eur J Prosthodont 2014;2:37-40. 